\title{
ALMOST PERIODIC FUNCTIONS AND HYPERBOLIC COUNTING
}

\author{
GIACOMO CHERUBINI
}

\begin{abstract}
We prove the existence of asymptotic moments and an estimate on the tails of the limiting distribution for a specific class of almost periodic functions. Then we introduce the hyperbolic circle problem, proving an estimate on the asymptotic variance of the remainder that improves a result of Chamizo. Applying the results of the first part we prove the existence of limiting distribution and asymptotic moments for three functions that are integrated versions of the remainder, and were considered originally (with due adaptations to our settings) by Wolfe, Phillips and Rudnick, and Hill and Parnovski.
\end{abstract}

\section{INTRODUCTION}

A classical problem in analytic number theory is that of determining if a given function, arising from a number theoretical question, admits a limiting distribution, and, similarly, if it admits finite asymptotic moments. As an example, consider the summatory von Mangoldt function

$$
\psi(x)=\sum_{1 \leq n \leq x} \Lambda(n) .
$$

It is an old result of Wintner [26] that, under the assumption of the Riemann hypothesis, the normalized remainder

$$
q(y)=\frac{\psi\left(e^{y}\right)-e^{y}}{e^{y / 2}}
$$

admits finite asymptotic moments of every order, and it has a limiting distribution. (the finiteness of the second moment had already been proved by Cramér [8]). Similarly, let $R(x)$ and $D(x)$ be the counting functions in the Gauss circle problem and the Dirichlet divisor problem, namely

$$
R(x)=\sum_{1 \leq n \leq x} r(n), \quad D(x)=\sum_{1 \leq n \leq x} d(n),
$$

where $r(n)$ is the number of ways of writing $n$ as a sum of two squares, and $d(n)$ the number of divisors of $n$. Then it is known that the normalized remainders

$$
u(y)=\frac{R\left(y^{2}\right)-\pi y^{2}}{y^{1 / 2}}, \quad v(y)=\frac{D\left(y^{2}\right)-\left(y^{2} \log y^{2}-(2 C-1) y^{2}\right)}{y^{1 / 2}}
$$

admit asymptotic moments of order $1 \leq k \leq 9$, and a limiting distribution (here $C$ is Euler's constant). The finiteness of the second moment is again a result of Cramér [9], the third and fourth moments can be found in the work of Tsang [24], while the existence of the moments of order up to nine, and of the limiting distribution, was proved by Heath-Brown [12, 13].

This paper is inspired by a recent article by Akbary, $\mathrm{Ng}$, and Shahabi [1], where the authors prove the existence of limiting distribution for a class of almost periodic

2010 Mathematics Subject Classification. Primary 11F72, 11P21, 42.30.

Key words and phrases. Hyperbolic lattice points, Almost periodic functions. 
functions. Here we prove a sufficient condition for the existence of asymptotic moments for a similar class of functions, complementing in this way Theorem 1.2 in [1].

We have in mind applications to counting problems in the hyperbolic plane (see $\S 1.1$ ), where also inputs from spectral theory of automorphic forms are needed. These problems turn out to be much harder than their classical analogues, due to the fact that we cannot exploit the spectral side in such an effective way as done in the classical setting. One of the reasons is that the spectrum of the euclidean Laplacian is completely explicit, but we do not know the exact location of the spectrum of the hyperbolic Laplacian. This complicates the proofs, and prevents us from proving results as good as in the classical case (at least with the same methods). Since the functions that appear in these problems share many similarities with more general almost periodic functions, it seems appropriate to prove the existence of limiting distribution and asymptotic moments in such a general framework.

The remainders in the prime number theorem, the Gauss circle problem, and the Dirichlet divisor problem, are suitable examples to describe the class of functions that we consider in Definition 1.1 below. Let $q(y)$ be the normalized remainder in the prime number theorem, as defined in (1.2). Assuming the Riemann hypothesis, there exist $y_{0}, X_{0}>0$ such that we can write (see e.g. chapter 17 in [10]), for $y \geq y_{0}$ and $X \geq X_{0}$,

$$
q(y)=-2 \Re\left(\sum_{0<\gamma \leq X} \frac{1}{\rho} e^{i \gamma y}\right)+O\left(\frac{e^{y / 2} \log \left(e^{y} X\right)}{X}+y e^{-y / 2}\right)
$$

where $\rho=1 / 2+i \gamma$ are the non-trivial zeros of the Riemann zeta function. For the Gauss circle problem (the Dirichlet divisor problem is similar), one can show that there exist $y_{0}, X_{0}>0$ such that for $y \geq y_{0}$ and $X \geq X_{0}$ we can write

$$
u(y)=2 \Re\left(\sum_{1 \leq n \leq X} \frac{r(n) e^{-i \pi / 4}}{2 \pi \sqrt{2} n^{3 / 4}} e^{4 \pi i y \sqrt{n}}\right)+O\left(\frac{X^{\varepsilon}}{y^{1 / 2}}+\frac{y^{\varepsilon}}{X^{1 / 2}}\right),
$$

For a reference see e.g. the book of Titchmarsh $[23,(12.4 .4)]$ or that of Ivić $[15$, Ch. 13]).

Identities (1.5) and (1.6) show that we can approximate the functions $q$ and $u$ by finite linear combinations of complex exponentials. The coefficients and the frequencies of such linear combinations depend on the problem.

Definition 1.1. Let $p \in \mathbb{R}, p \geq 1$, and let $\phi:[0, \infty) \rightarrow \mathbb{R}$. We say that $\phi$ is a $p$-function if there exist a strictly increasing sequence $\left\{\lambda_{n}\right\}$ of positive real numbers tending to infinity, a sequence of complex numbers $\left\{r_{n}\right\}$, and numbers $y_{0}, X_{0}>0$, such that $\phi \in L^{p}\left(\left[0, y_{0}\right]\right)$, and for $y \geq y_{0}, X \geq X_{0}$, we have

$$
\phi(y)=2 \Re\left(\sum_{0<\lambda_{n} \leq X} r_{n} e^{i \lambda_{n} y}\right)+\mathcal{E}(y, X),
$$

where $\mathcal{E}(y, X)$ satisfies

$$
\lim _{Y \rightarrow \infty} \frac{1}{Y} \int_{y_{0}}^{Y}|\mathcal{E}(y, X(Y))|^{p} d y=0
$$

for some non-decreasing function $X(Y)$ tending to infinity. We say moreover that $\phi$ is a $(p, \beta)$-function if $\phi$ is a $p$-function, and there exists $\beta \in \mathbb{R}$ such that

$$
\sum_{T \leq \lambda_{n} \leq T+1}\left|r_{n}\right| \ll \frac{1}{T^{\beta}} \text { for } T \gg 1
$$

Remark 1.2. It follows from [1, Th. 1.2] that a $(p, \beta)$-function $\phi$ such that $p \geq 2$ and $\beta>1 / 2$ is a $B^{2}$-almost periodic function. Definition 1.1 is slightly more 
general, as we can consider $1 \leq p<2$ and $\beta \leq 1 / 2$. However, in Theorem 1.3, Theorem 1.10, and Corollary 1.14 below, one should keep in mind that the results concern almost periodic functions as soon as $p \geq 2$ and $\beta>1 / 2$.

The following Theorem 1.3 gives a sufficient condition to ensure that a $(p, \beta)$ function admits finite asymptotic moments. The condition

$$
\limsup _{Y \rightarrow \infty} \sup _{y \in\left[y_{0}, Y\right]}|\mathcal{E}(y, X(Y))|=0
$$

is required to cover some cases where (1.8) does not suffice. Observe that (1.10) implies (1.8), for any $p \geq 1$, since we have

$$
\frac{1}{Y} \int_{y_{0}}^{Y}|\mathcal{E}(y, X(Y))|^{p} d y \leq \sup _{y \in\left[y_{0}, Y\right]}|\mathcal{E}(y, X(Y))|^{p} .
$$

Theorem 1.3. Let $p \in \mathbb{N}$, and let $\phi$ be a $(p, \beta)$-function with $\beta>1-1 / p$. If $p$ is odd and greater than 1, assume that (1.10) holds. Then the asymptotic moment

$$
L_{n}=\lim _{Y \rightarrow \infty} \frac{1}{Y} \int_{0}^{Y} \phi(y)^{n} d y
$$

exists, for every $1 \leq n \leq p$.

Remark 1.4. It is easy to see that the function $q(y)$ in (1.5) (under RH), and the function $u(y)$ in (1.6), are $p$-functions for every $p \geq 1$ (by choosing $X(Y)=e^{Y}$ in the first case, and $X(Y)=Y$ in the second). Because of the asymptotic formula for $N(T)$, the number of non-trivial zeros of the Riemann zeta function with imaginary part in $(0, T]$, we have (see Davenport [10, p. 59])

$$
N(T)=\frac{T}{2 \pi} \log \frac{T}{2 \pi}-\frac{T}{2 \pi}+O(\log T),
$$

and in view of the standard asymptotic formula

$$
\sum_{1 \leq n \leq x} r(n)=\pi x+O(\sqrt{x})
$$

we conclude that we have

$$
\sum_{\substack{\rho=1 / 2+i \gamma: \\ T \leq \gamma \leq T+1}} \frac{1}{|\rho|} \ll \frac{\log T}{T} \text { and } \sum_{T \leq \sqrt{n} \leq T+1} \frac{r(n)}{n^{3 / 4}} \ll \frac{1}{T^{1 / 2}} .
$$

This shows that the function $q$ is (under $\mathrm{RH}$ ) a $(p, \beta)$-function for $p \geq 1$ and any $\beta<1$, whereas the function $u$ is a $(p, \beta)$-function for $p \geq 1$ and $\beta=1 / 2$.

Remark 1.5. Notice that if $\phi$ is a $(p, \beta)$-function, then it is also a $\left(p^{\prime}, \beta^{\prime}\right)$-function, for every $p^{\prime} \leq p$ and $\beta^{\prime} \leq \beta$. It is thus interesting to find the largest $p$ and $\beta$ such that $\phi$ is a $(p, \beta)$-function: the pair $(p, \beta)$ gives, by Theorem 1.3 , the largest range for which we can prove finiteness of the moments of $\phi$.

Remark 1.6. The existence of the second moment was proved by Akbary et al. [1, Th. 1.14] for $(2, \beta)$-functions with $\beta>1 / 2$, as a corollary of the fact that such functions are Besicovitch $B^{2}$-almost periodic. Theorem 1.3 extends their result to moments of higher order.

Remark 1.7. We give an expression for $L_{n}$ in (2.7), with notation introduced in section 2. The value of the moments is obtained as the sum of an absolutely convergent series that involves the data from the sequence of the frequencies $\lambda_{n}$ and from the coefficients $r_{n}$. 
Remark 1.8. If $\phi$ is a $(p, \beta)$-function for $p$ arbitrarily large and every $\beta<1$, then Theorem 1.3 implies that all the moments of $\phi$ exist. This is the case of the remainder $q(y)$ in the prime number theorem (under $\mathrm{RH}$ ).

On the other hand, when $\beta \leq 1 / 2$, an extra input must be given in order to show that the second and higher moments exist. This is what happens for the remainder $u(y)$ in the Gauss circle problem, where additional properties of the problem are used to prove the existence of asymptotic variance and moments of order $1 \leq n \leq 9$. Indeed, if $\phi$ is a $(p, \beta)$-function with $\beta \leq 1 / 2$, then it does not follow from [1, Th. $1.2]$ that $\phi$ is an almost periodic function, and some extra information must be used to show if this is the case.

We consider the cases $\beta=1 / 2$ and $\beta=1$ as "extremal", so that Theorem 1.3 covers the intermediate situation when $\beta$ is some number strictly between $1 / 2$ and 1 .

Remark 1.9. Consider an irreducible unitary cuspidal automorphic representation $\pi$ of $\mathrm{GL}_{d}\left(\mathbb{A}_{\mathbb{Q}}\right)$, and let $L(s, \pi)$ be the automorphic $L$-function attached to $\pi$.

Let $\psi(x, \pi)$ be the prime counting function associated to $L(s, \pi), M(x, \pi)$ the main term in the asymptotic expansion of $\psi(x, \pi)$, and denote the remainder by $E(x, \pi)$. Assuming the corresponding Riemann hypothesis for $L(s, \pi)$, the function

$$
e^{-y / 2} E\left(e^{y}, \pi\right)
$$

has the structure of a $(p, \beta)$-function for $p \geq 1$ and any $\beta<1$ (see [1, Proposition 4.2]). Theorem 1.3 shows that $e^{-y / 2} E\left(e^{y}, \pi\right)$ admits finite moments of every order. The result should be compared with [1, Cor. 1.15], where the existence of the variance is proved.

If we consider a $(p, \beta)$-function $\phi$ such that $p \geq 2$ and $\beta>1 / 2$, it follows from [1, Th. 1.2] that $\phi$ admits a limiting distribution. Our second result concerns the tails of the limiting distribution.

Theorem 1.10. Let $\phi$ be $a(p, \beta)$-function with $p \geq 2$ and $\beta>1 / 2$. Then $\phi$ admits a limiting distribution $\mu$ with tails of size

$$
\mu((-\infty,-S] \cup[S,+\infty)) \ll S^{-(2 \beta-1) /(2-2 \beta)} .
$$

For $\beta=1$ we have exponential decay, that is, there exists a positive constant $c_{\phi}>0$ such that $\mu((-\infty,-S] \cup[S,+\infty)) \ll \exp \left(-c_{\phi} S\right)$. For $\beta>1$ the measure $\mu$ is compactly supported.

Remark 1.11. The proof of the existence of the measure $\mu$ can be found in several papers (see e.g. [12, 2, 21, 1]). The estimate on the tails for general $\beta$ does not seem however to appear in these papers. In section 3 we prove Theorem 1.10, following the argument of [21, p. 178-181], which easily generalizes to (1.12).

Remark 1.12. In the case of the prime number theorem and $L$-functions discussed in [21], the remainder terms have an almost periodic expansion with corresponding coefficients $r_{n}$ that satisfy

$$
\sum_{T \leq \lambda_{n} \leq T+1}\left|r_{n}\right| \ll \frac{\log T}{T} .
$$

This leads to exponential decay of type $O(\exp (-c \sqrt{\lambda}))$ for the tails of the limiting distributions. Similarly, a bound in (1.13) of type $O\left(T^{-1} \log ^{m}(T)\right), m \geq 0$, leads to an upper bound for the tails of $\mu$ of type $O\left(\exp \left(-c \lambda^{1 /(m+1)}\right)\right)$.

Remark 1.13. Lower bounds for the tails of the limiting distribution $\mu$ of a general $(p, \beta)$-function $\phi$ can also be proved by similar argument as in [21], but we have decided not to discuss them here. Moreover, assuming the extra condition that the frequencies $\lambda_{n}$ are linearly independent, a much stronger decay on the tails of $\mu$ 
can be proved, and one can show that the Fourier transform of $\mu$ can be expressed in terms of Bessel functions (see [1, Th. 1.9] and also [18, 19, 21]).

If a function $\phi$ admits a limiting distribution $\mu$ and finite asymptotic moments, a natural question to ask is whether the moments of $\phi$ coincide with the moments of the distribution $\mu$. This can be a nontrivial question, as there exist functions for which the moments do not agree (for instance if the function has very rare but very large peaks).

Due to the estimate on the tails of the limiting distribution provided by Theorem 1.10, we can prove the following corollary, where we show that up to a certain order the moments of a $(p, \beta)$-function are indeed equal to those of its limiting distribution.

Corollary 1.14. Let $\phi$ be $a(p, \beta)$-function with $p \geq 2$ and $\beta>1-1 /(2 p+4)$. Then the moments of $\phi$ of order $1 \leq n \leq p$ coincide with the moments of its limiting distribution $\mu$. In other words, for $1 \leq n \leq p$ we have

$$
\lim _{Y \rightarrow \infty} \frac{1}{Y} \int_{0}^{Y} \phi(y)^{n} d y=\int_{\mathbb{R}} x^{n} d \mu .
$$

1.1. Applications to the hyperbolic circle problem. In the second part of the paper we apply the above results to the hyperbolic lattice point counting problem, which is defined as follows. For $\Gamma \leq \operatorname{PSL}(2, \mathbb{R})$ a cofinite Fuchsian group and $z, w \in \mathbb{H}$, define the function

$$
N(s, z, w)=\{\gamma \in \Gamma: d(z, \gamma w) \leq s\},
$$

where $d$ is the hyperbolic distance. The function $N(s, z, w)$ counts the number of translates of the point $w$ by elements $\gamma$ of the group $\Gamma$ that fall inside the hyperbolic ball $B(z, s)$ of center $z$ and radius $s$.

Spectral theory of automorphic forms provides the main asymptotic of $N(s, z, w)$ as $s$ tends to infinity, as well as a finite number of secondary terms (associated to the small eigenvalues), that we collect in the "complete main term" $M(s, z, w)$, see (4.23). The remainder in the problem is then defined as

$$
E(s, z, w)=N(s, z, w)-M(s, z, w) .
$$

In section 4 we introduce the notation and technical estimates related to the problem, and we prove an upper bound on the asymptotic variance for the normalized remainder term $e(s, z, w):=e^{-s / 2} E(s, z, w)$ as follows.

Theorem 1.15. Let $\Gamma$ be a cofinite Fuchsian group, let $z, w \in \mathbb{H}$, and let $T \gg 1$. Then

$$
\int_{T}^{T+1}|e(s, z, w)|^{2} d s \ll T .
$$

Remark 1.16. Theorem 1.15 improves on a result by Chamizo [5, Cor. 2.1.1] that corresponds, in our notation, to an upper bound $O\left(T^{2}\right)$ in (1.17). The method of proof is different: Chamizo's proof uses the large sieve in Riemann surfaces [4], while we directly integrate in the pretrace formula for $e(s, z, w)$. This strategy was suggested in [3, p. 27]. Moreover, differently from Chamizo, we include the contribution associated to the eigenvalue $\lambda=1 / 4$ in the main term $M(s, z, w)$.

Remark 1.17. The problem of the finiteness of the asymptotic second moment of $e(s, z, z)$ was already discussed by Phillips and Rudnick [20]. Like us, they were not able to show that the second moment of the normalized remainder is finite. On the other hand, they show that the limit

$$
\lim _{T \rightarrow \infty} \frac{1}{T} \int_{0}^{T}|e(s, z, z)|^{2} d s
$$


is non-zero, and they provide numerics that suggest that the limit should exist.

Remark 1.18. Theorem 1.15 shows that the asymptotic variance of $e(s, z, w)$, if not finite, diverges at most linearly with $T$. Also, it shows that the function $E(s, z, w)$ is "on average" bounded by $O\left(\sqrt{s} e^{s / 2}\right)$, which is consistent with the conjectural bound

$$
E(s, z, w)=O\left(e^{s(1 / 2+\varepsilon)}\right), \quad \forall \varepsilon>0 .
$$

A straightforward consequence of Theorem 1.15 is that for every $\alpha>0$ the set

$$
\left\{s \geq 0:|E(s, z, w)|>s^{1+\alpha} e^{s / 2}\right\}
$$

has finite Lebesgue measure, hence $E(s, z, w)$ violates the conjectural bound at most in a set of finite measure.

We move then to consider certain integrated versions of $E(s, z, w)$. We start by defining, for $\Gamma$ cocompact, $z \in \Gamma \backslash \mathbb{H}$, and $s \geq 0$, the following functions:

$$
\begin{gathered}
G_{1}(s, z):=\frac{1}{e^{s}} \int_{\Gamma \backslash \mathbb{H}}|E(s, z, w)|^{2} d \mu(w), \\
G_{2}(s):=\frac{1}{e^{s}} \iint_{\Gamma \backslash \mathbb{H} \times \Gamma \backslash \mathbb{H}}|E(s, z, w)|^{2} d \mu(z) d \mu(w) .
\end{gathered}
$$

The functions $G_{1}, G_{2}$ are thus defined by integrating away one (resp. both) space variable. For $\Gamma$ cocompact and $z \in \Gamma \backslash \mathbb{H}, s \geq 0$, we define also

$$
G_{3}(s, z):=\frac{1}{e^{s / 2}} \int_{0}^{s} E(x, z, z) d x,
$$

which gives the integral of $E(s, z, z)$ in the radial variable. Applying the results of the first part of the paper we obtain the following theorem.

Theorem 1.19. Let $\Gamma$ be cocompact, $z \in \Gamma \backslash \mathbb{H}$, and let $s \geq 0$. Then for $i=1,2,3$ the function $G_{i}$ is bounded in $s$, admits moments of every order, limiting distribution $\mu_{i}$ of compact support, and the moments of $G_{i}$ coincide with the moments of $\mu_{i}$.

Remark 1.20. Notice that, since the functions $G_{1}$ and $G_{3}$ depend on $z$, the limiting distributions $\mu_{1}$ and $\mu_{3}$ will also depend on $z$.

Remark 1.21. The function $G_{1}$ was considered by Hill and Parnovski [14], who only proved that $G_{1}$ is bounded. The integration of both space variables that defines $G_{2}$ was studied by Wolfe [27], who again studied its pointwise behaviour but did not have the distributional result. The function $G_{3}$ was studied by Phillips and Rudnick [20] in relation to the proof that the asymptotic mean of $E(s, z, z)$ vanishes. They also consider cofinite groups that are not cocompact, and it is probably possible to extend the proof for $G_{3}$ to the general cofinite case, but we have refrained from doing this here.

Remark 1.22. Whereas we cannot prove that the remainder $e(s, z, z)$ admits finite variance, we see that the function $G_{3}(s, z)$ admits not only finite variance, but finite moments of every order. In [7] Cherubini and Risager considered integration of $e(s, z, z)$ to fractional order $0<\alpha<1$, and showed that the resulting function $e_{\alpha}(s, z)$, has finite asymptotic variance for every $0<\alpha<1$. This means that not a full integration, but integration to any positive small order suffices to give finite second moment. From the variance of $e_{\alpha}(s, z)$ one might then expect to recover, in the limit as $\alpha$ tends to 0 , the variance of $e(s, z, z)$, but it seems hard to prove this. We refer to $[7, \S 8]$ for a more detailed discussion on the subject. 


\section{Asymptotic moments}

In this section we prove Theorem 1.3. Let $\phi$ be a $(p, \beta)$-function. Set $\lambda_{-n}=-\lambda_{n}$ and $r_{-n}=\bar{r}_{n}$, and define the function

$$
S(y, X):=\sum_{\substack{n \in \mathbb{Z}: x \\\left|\lambda_{n}\right| \leq X}} r_{n} e^{i \lambda_{n} y},
$$

so that we have, for $y \geq y_{0}$ and $X \geq X_{0}$, the identity

$$
\phi(y)=S(y, X)+\mathcal{E}(y, X) .
$$

In view of this relation and of assumption (1.8), we expect that the moments of $\phi$ can be computed simply by looking at the moments of $S(y, X)$.

On taking the $n$-th power we can write

$$
S(y, X)^{n}=\sum_{\substack{J \in \mathbb{Z}^{n}: \\\left|\lambda_{J}\right| \leq X}} A\left(r_{J}\right) e^{i y \vartheta\left(\lambda_{J}\right)},
$$

where $J=\left(j_{1}, \ldots, j_{n}\right)$ is a multiindex, $\lambda_{J}=\left(\lambda_{j_{1}}, \ldots, \lambda_{j_{n}}\right)\left(\right.$ similarly $\left.r_{J}=\left(r_{j_{1}}, \ldots, r_{j_{n}}\right)\right)$, $\left|\lambda_{J}\right| \leq X$ means that all entries are smaller than $X$, and

$$
A\left(r_{J}\right)=\prod_{k=1}^{n} r_{j_{k}}, \quad \vartheta\left(\lambda_{J}\right)=\sum_{k=1}^{n} \lambda_{j_{k}} .
$$

Note that the sum in (2.2) runs over $J \in \mathbb{Z}^{n}$; however, in order to simplify notation, we will omit the condition $J \in \mathbb{Z}^{n}$, and only write $\left|\lambda_{J}\right| \leq X$, throughout the rest of this section. Setting $X=X(Y)$ and integrating in $y$ we get

$$
\frac{1}{Y} \int_{y_{0}}^{Y} S(y, X(Y))^{n} d y=\sum_{\left|\lambda_{J}\right| \leq X(Y)} A\left(r_{J}\right) \frac{1}{Y} \int_{y_{0}}^{Y} e^{i y \vartheta\left(\lambda_{J}\right)} d y .
$$

The integral equals $\left(Y-y_{0}\right)$ when $\vartheta\left(\lambda_{J}\right)=0$, and otherwise we can bound

$$
\left|\frac{1}{Y} \int_{y_{0}}^{Y} e^{i y \vartheta_{g}\left(\lambda_{J}\right)} d y\right| \leq \frac{4}{1+Y\left|\vartheta_{g}\left(\lambda_{J}\right)\right|} .
$$

We define the sets (diagonal and off-diagonal)

$$
\begin{aligned}
& \mathscr{D}^{Y}:=\left\{\left|\lambda_{J}\right| \leq X(Y) \mid \vartheta\left(\lambda_{J}\right)=0\right\} \\
& \mathscr{O}^{Y}:=\left\{\left|\lambda_{J}\right| \leq X(Y) \mid \vartheta\left(\lambda_{J}\right) \neq 0\right\} .
\end{aligned}
$$

The contribution of the off-diagonal is negligible if we assume that the coefficients $r_{n}$ decay sufficiently fast, and we have the following.

Proposition 2.1. Let $S(y, X)$ be as in (2.1), and assume that (1.9) holds with $\beta>1-1 / n$. Then the limit

$$
\lim _{Y \rightarrow \infty} \frac{1}{Y} \int_{y_{0}}^{Y} S(y, X(Y))^{n} d y
$$

exists, and is given by

$$
\sum_{\lambda_{J} \in \mathscr{D}^{\infty}} A\left(r_{J}\right),
$$

where $A\left(r_{J}\right)$ is defined in (2.3). The sum in (2.7) is absolutely convergent, as shown at the end of the proof. 
Proof. From (2.4) and (2.5) we can write

$$
\begin{aligned}
\frac{1}{Y} \int_{y_{0}}^{Y} S(y, X(Y))^{n} d y & =\sum_{\lambda_{J} \in \mathscr{D}^{Y}} A\left(r_{J}\right)\left(1+O\left(y_{0} Y^{-1}\right)\right) \\
& +O\left(\sum_{\lambda_{J} \in \mathscr{O}^{Y}} \frac{\left|A\left(r_{J}\right)\right|}{1+Y\left|\vartheta\left(\lambda_{J}\right)\right|}\right) .
\end{aligned}
$$

We bound the off-diagonal first. Let $\mathscr{O}$ denote the sum on the second line of (2.8). If we can show that the sum is bounded for every $Y \geq 1$, then by Lebesgue dominated convergence we will conclude that the sum vanishes as $Y \rightarrow \infty$. Ignoring the $\lambda_{j}<1$ and grouping the terms corresponding to $a_{i} \leq \lambda_{j} \leq a_{i}+1, a_{i} \in \mathbb{Z}^{+}$, because of (1.9) we have

$$
\mathscr{O} \ll \sum_{s=0}^{n} \sum_{k=1}^{\infty} \sum_{l=1}^{\infty} \frac{B_{s}[k] B_{n-s}[l]}{1+|k-l+O(n)|} \quad \text { where } \quad B_{r}[m]=\sum_{a_{1}+\cdots+a_{r}=m}\left(a_{1} \cdots a_{r}\right)^{-\beta} .
$$

It can be proved that $B_{r}[m] \ll_{r} m^{(1-\beta) r-1}$, for instance by induction on $r$,

$$
B_{r}[m] \ll \sum_{a_{r} \leq m} B_{r-1}[m] \ll m^{1-\beta} m^{(1-\beta)(r-1)-1}=m^{(1-\beta) r-1} .
$$

We have $1+|k-l+O(n)| \gg_{n}|k-l|$, so

$$
\mathscr{O} \ll \sum_{k \neq l} \frac{1}{k^{u} l^{v}|k-l|} \quad \text { with } \quad \begin{aligned}
& u=(\beta-1) s+1 \\
& v=(\beta-1)(n-s)+1 .
\end{aligned}
$$

Since $\beta>1-1 / n$, splitting the summation for $1 \leq k \leq l / 2$ and $l / 2<k<l$ (and similarly for $l$ when $l<k$ ) we can upper bound (2.9) by convergent harmonic series $\sum_{k \geq 1} k^{-1-\varepsilon}$ so that we conclude that $\mathscr{O}$ is bounded for every $Y \geq 1$.

Let $\mathscr{D}$ denote the sum appearing in the first line in (2.8). We show that this sum is absolutely convergent for every $Y \geq 1$. Indeed, we can bound

$$
|\mathscr{D}| \ll \sum_{s=0}^{n} \sum_{k=1}^{\infty} B_{s}[k] B_{n-s}[l]
$$

with $|l-k| \leq n$, and using $B_{r}[m] \ll m^{(1-\beta) r-1}$ we obtain

$$
|\mathscr{D}| \ll \sum_{k=1}^{\infty} \frac{1}{k^{u+v}}
$$

which converges since $u+v>1$. For $Y \rightarrow \infty$, we see that the sum in (2.7) is absolutely convergent.

Proof of Theorem 1.3. Let us prove that for every $1 \leq n \leq p$ we have

$$
\lim _{Y \rightarrow \infty} \frac{1}{Y} \int_{0}^{Y} \phi(y)^{n} d y=\lim _{Y \rightarrow \infty} \frac{1}{Y} \int_{y_{0}}^{Y} S(y, X(Y))^{n} d y
$$

The result will then follow from Proposition 2.1. Consider the case $n=p$, and assume first that $p$ is even; from Proposition 2.1 we know that the $p$-th moment of $S$ is finite. Hence we can write, using Hölder's inequality,

$$
\begin{aligned}
& \frac{1}{Y} \int_{0}^{Y} \phi(y)^{p} d y=\frac{1}{Y} \int_{y_{0}}^{Y} S(y, X(Y))^{p} d y+O\left(\frac{1}{Y} \int_{0}^{y_{0}}|\phi(y)|^{p} d y\right) \\
+ & \left(\sum_{n=0}^{p-1} \frac{1}{Y}\left(\int_{y_{0}}^{Y}|S(y, X(Y))|^{p} d y\right)^{\frac{n}{p}}\left(\int_{y_{0}}^{Y}|\mathcal{E}(y, X(Y))|^{p} d y\right)^{\frac{p-n}{p}}\right) .
\end{aligned}
$$


Because of (1.8), the errors in (2.11) tend to zero as $Y \rightarrow \infty$, and we obtain (2.10). Assume now that $p$ is odd. Then we can write

$$
\begin{aligned}
\frac{1}{Y} \int_{0}^{Y} \phi(y)^{p} d y & =\frac{1}{Y} \int_{y_{0}}^{Y} S(y, X(Y))^{p} d y+O\left(\frac{1}{Y} \int_{0}^{y_{0}}|\phi(y)|^{p} d y\right) \\
& +O\left(\sum_{n=0}^{p-1} \frac{1}{Y} \int_{y_{0}}^{Y}|S(y, X(Y))|^{n}|\mathcal{E}(y, X(Y))|^{p-n} d y\right) .
\end{aligned}
$$

If $p=1$ then the error contains only one term, and this tends to zero as $Y \rightarrow \infty$, in view of (1.8), and we obtain (2.10). For $p>1$, we cannot use Hölder inequality exactly in the same way as in (2.12) because we cannot bound by $O(1)$ the $p$-th moment of $|S|$ (we can do this without absolute value), and we argue a little different, by bounding $\mathcal{E}$ by its absolute value. Proposition 2.1 gives $Y^{-1} \int_{y_{0}}^{Y}|S(y, X(Y))|^{n} d y \ll 1$ for every $1 \leq n \leq p-1$, and condition (1.10) shows that in the limit as $Y \rightarrow \infty$ the parenthesis tends to zero, thus we obtain (2.10). For $n \leq p-1$ the same argument as in (2.11)-(2.12) works. This proves the theorem.

\section{Limiting Distribution}

In this section we prove Theorem 1.10. We start by recalling the definition of limiting distribution.

Definition 3.1. A limiting distribution for a function $\phi:[0, \infty) \rightarrow \mathbb{R}$ is a probability measure $\mu$ on $\mathbb{R}$ such that the limit

$$
\lim _{Y \rightarrow \infty} \frac{1}{Y} \int_{0}^{Y} g(\phi(y)) d y=\int_{\mathbb{R}} g d \mu
$$

holds for every bounded continuous function $g$ on $\mathbb{R}$.

We give now two preparatory lemmata. Let $X>T>2$ and consider the functions

$$
\phi_{T}(y)=2 \Re\left(\sum_{0<\lambda_{n} \leq T} r_{n} e^{i \lambda_{n} y}\right), \quad \psi_{T}(y, X)=2 \Re\left(\sum_{T<\lambda_{n} \leq X} r_{n} e^{i \lambda_{n} y}\right)+\mathcal{E}(y, X) .
$$

Lemma 3.2. Assume the hypothesis of Theorem 1.10. Then for $Y, T \gg 1$ with $X(Y) \geq T$, we have

$$
\frac{1}{Y} \int_{y_{0}}^{Y}\left|\psi_{T}(y, X(Y))\right|^{2} d y \ll \frac{1}{T^{2 \beta-1}} .
$$

Proof. This follows from [17, Lemma 7.1] and (1.9).

Lemma 3.3. For each $T \geq 2$ there exists a probability measure $\nu_{T}$ on $\mathbb{R}$ such that

$$
\nu_{T}(f):=\int_{\mathbb{R}} f(x) d \nu_{T}(x)=\lim _{Y \rightarrow \infty} \frac{1}{Y} \int_{y_{0}}^{Y} f\left(\phi_{T}(y)\right) d y
$$

for every bounded Lipschitz continuous function $f$ on $\mathbb{R}$. In addition, there is a constant $c>0$ such that the support of $\nu_{T}$ lies in the ball $B\left(0, c T^{1-\beta}\right)$ for $\beta<$ 1 , respectively $B(0, c \log T)$ for $\beta=1$. For $\beta>1$ the support of $\nu_{T}$ is bounded independently of $T$.

Proof. The existence of the measure is [21, Lemma 2.3]. The statement about the support of $\nu_{T}$ follows from the fact that

$$
\left|\phi_{T}(y)\right| \ll T^{1-\beta}, \quad\left|\phi_{T}(y)\right| \ll \log T,
$$

respectively for $\beta<1, \beta=1$, and $\left|\phi_{T}(y)\right| \ll 1$ independently of $T$ for $\beta>1$. 
Proof of Theorem 1.10. We follow closely [21, 179-181]. Consider a bounded Lipschitz continuous function $f$, with Lipschitz constant $c_{f}$, so that

$$
|f(x)-f(y)| \leq c_{f}|x-y| \text {. }
$$

Then we have for $Y \gg 1$

$$
\frac{1}{Y} \int_{y_{0}}^{Y}\left(f(\phi(y))-f\left(\phi_{T}(y)\right)\right) d y \ll\left(\frac{1}{Y} \int_{y_{0}}^{Y}\left|\psi_{T}(y, X(Y))\right|^{2} d y\right)^{1 / 2} \ll \frac{1}{T^{\beta-1 / 2}},
$$

so that taking the limit as $Y \rightarrow \infty$ we obtain

$$
\begin{aligned}
\nu_{T}(f)-O\left(\frac{1}{T^{\beta-1 / 2}}\right) & \leq \liminf _{Y \rightarrow \infty} \frac{1}{Y} \int_{y_{0}}^{Y} f(\phi(y)) d y \\
& \leq \limsup _{Y \rightarrow \infty} \frac{1}{Y} \int_{y_{0}}^{Y} f(\phi(y)) d y \leq \nu_{T}(f)+O\left(\frac{1}{T^{\beta-1 / 2}}\right) .
\end{aligned}
$$

Since $T$ can be arbitrarily large, we conclude that the lim inf and lim sup coincide, i.e. that

$$
\mu(f):=\lim _{Y \rightarrow \infty} \frac{1}{Y} \int_{y_{0}}^{Y} f(\phi(y)) d y
$$

exists. Thus there exists a Borel measure $\mu$ on $\mathbb{R}$ such that (3.3) holds for all $f$ satisfying (3.2). Moreover, for such $f$,

$$
\left|\mu(f)-\nu_{T}(f)\right| \ll \frac{1}{T^{\beta-1 / 2}} .
$$

In view of Lemma 3.3 and (3.4) we also have for $\beta<1$

$$
\mu\left(B_{\lambda}^{c}\right)=\nu_{T}\left(B_{\lambda}^{c}\right)+O\left(\frac{1}{T^{\beta-1 / 2}}\right)=O\left(\frac{1}{T^{\beta-1 / 2}}\right)
$$

for $\lambda=c T^{1-\beta}\left(B_{\lambda}^{c}\right.$ is the complement of the open ball of radius $\left.\lambda\right)$. This leads to

$$
\mu\left(B_{\lambda}^{c}\right)=O\left(\lambda^{-(2 \beta-1) /(2-2 \beta)}\right) .
$$

In the case when $\beta=1$ we insert $\lambda=c \log T$ in (3.5), which gives $\mu\left(B_{\lambda}^{c}\right)=$ $O\left(e^{-\lambda / 2 c}\right)$. Finally, for $\beta>1$, the compactness of the support of $\mu$ follows from the fact that $\phi_{T}$ is bounded independently of $T$.

Proof of Corollary 1.14. The proof follows the lines of [11, Lemma 2.5]. We show that (1.14) holds for $n=p$, as the case for $n<p$ is similar. First observe that by Theorem 1.3 we have the bound as $Y \rightarrow \infty$

$$
\frac{1}{Y} \int_{0}^{Y}|\phi(y)|^{p} d y \ll 1
$$

Consider for $S \gg 1$ the Lipschitz bounded continuous function

$$
H_{S}(x):= \begin{cases}0 & \text { if }|x| \leq S \\ |x|-S & \text { if } S<|x| \leq S+1, \\ 1 & \text { if }|x|>S+1\end{cases}
$$

By Theorem 1.10 we have

$$
\lim _{Y \rightarrow \infty} \frac{1}{Y} \int_{0}^{Y} H_{S}(\phi(y)) d y=\int_{\mathbb{R}} H_{S}(x) d \mu \ll S^{-(2 \beta-1) /(2-2 \beta)} .
$$

It follows that

$$
\limsup _{Y \rightarrow \infty} \frac{1}{Y} \int_{\substack{0 \leq y \leq Y \\|\phi(y)| \geq S+1}} d R \leq \limsup _{Y \rightarrow \infty} \frac{1}{Y} \int_{0}^{Y} H_{S}(\phi(y)) d y \ll S^{-(2 \beta-1) /(2-2 \beta)} .
$$


In view of the bound (3.6) we can write

$$
\begin{aligned}
\limsup _{Y \rightarrow \infty} \frac{1}{Y} \int_{\substack{0 \leq y \leq Y \\
|\phi(y)| \geq S}}|\phi(y)|^{p} d y & =\limsup _{Y \rightarrow \infty} \sum_{\ell=0}^{\infty} \frac{1}{Y} \int_{\substack{0 \leq y \leq Y \\
|\phi(y)| \geq S+\ell}}|\phi(y)|^{p} d y \\
& \ll \sum_{\ell=0}^{\infty}(S+\ell+1)^{p}(S+\ell)^{-(2 \beta-1) /(2-2 \beta)} \\
& \ll S^{p+1-(2 \beta-1) /(2-2 \beta)} .
\end{aligned}
$$

Here we used (3.6) to interchange the limsup with the infinite series, and the fact that $\beta>1-1 /(2 p+4)$ to estimate the sum of the series. Define now the bounded Lipschitz continuous function

$$
G_{S}(x):= \begin{cases}x^{p} & 0 \leq x \leq S \\ S^{p}(S+1-x) & S<x \leq S+1, \\ 0 & x>S+1\end{cases}
$$

for $x \geq 0$, and $G_{S}(-x)=(-1)^{p} G_{S}(x)$ for $x<0$. We obtain

$$
\begin{aligned}
\limsup _{Y \rightarrow \infty} \frac{1}{Y} \int_{0}^{Y} \phi(y)^{p} d y & =\limsup _{Y \rightarrow \infty} \frac{1}{Y} \int_{0}^{Y} G_{S}(\phi(y)) d y \\
& +O\left(S^{p+1-(2 \beta-1) /(2-2 \beta)}\right) \\
= & \int_{\mathbb{R}} G_{S}(x) d \mu+O\left(S^{p+1-(2 \beta-1) /(2-2 \beta)}\right) \\
= & \int_{\mathbb{R}} x^{p} d \mu+O\left(S^{p+1-(2 \beta-1) /(2-2 \beta)}\right) .
\end{aligned}
$$

Taking $S \rightarrow \infty$ we conclude that

$$
\limsup _{Y \rightarrow \infty} \frac{1}{Y} \int_{0}^{Y} \phi(y)^{p} d y=\int_{\mathbb{R}} x^{p} d \mu .
$$

A similar argument works for the liminf, and this concludes the proof.

\section{Applications to hyperbolic counting}

In this section we prove Theorem 1.15. The strategy of proof consists in applying the pretrace formula [16, Th. 7.4] to a regularized version of the automorphic kernel

$$
N(s, z, w)=\sum_{\gamma \in \Gamma} \mathbf{1}_{[0, s]}(d(z, \gamma w))=\sum_{\gamma \in \Gamma} \mathbf{1}_{[0,(\cosh s-1) / 2]}(u(z, \gamma w)) .
$$

We construct here the smoothing and explain how the "complete" main term $M(s, z, w)$ is defined both for the sharp and for the regularized problem (see (4.23) and (4.24)).

Let $\delta$ be a small positive real number, $0<\delta<1$, and consider the function

$$
k_{\delta}(u):=\frac{1}{4 \pi \sinh ^{2}(\delta / 2)} \mathbf{1}_{[0,(\cosh (\delta)-1) / 2]}(u)
$$

where $\mathbf{1}_{[0, A]}$ is the indicator function of the set $[0, A]$. For $u=u(z, w)$, the function $k_{\delta}$ is the indicator function of a ball of radius $\delta$ in the hyperbolic plane, normalized so that the ball has unit volume. In other words, it satisfies

$$
\int_{\mathbb{H}} k_{\delta}(u(z, w)) d \mu(z)=1
$$

Define $k^{ \pm}(u)$ as the functions given by the convolution product

$$
k^{ \pm}(u):=\left(\mathbf{1}_{[0,(\cosh (s \pm \delta)-1) / 2]} * k_{\delta}\right)(u),
$$


where the hyperbolic convolution of two functions $k_{1}, k_{2}$ is defined $[5,(2.11)]$ as

$$
\int_{\mathbb{H}} k_{1}(u(z, v)) k_{2}(u(v, w)) d \mu(v)
$$

Notice that the convolution of $k_{1}$ and $k_{2}$ in (4.3) only depends on the distance between $z$ and $w$, and thus defines a function of $u=u(z, w)$, as written in compact form in (4.2). Because of the triangle inequality $d(z, w) \leq d(z, v)+d(v, w)$, for $Z \geq 0$ the convolution $\mathbf{1}_{[0, \cosh (Z)-1) / 2]} * k_{\delta}$ satisfies

$$
\left(\mathbf{1}_{[0, \cosh (Z)-1) / 2]} * k_{\delta}\right)(u(z, w))= \begin{cases}1 & d(z, w) \leq Z-\delta \\ 0 & d(z, w) \geq Z+\delta .\end{cases}
$$

From this we deduce that

$$
k^{-}(u) \leq \mathbf{1}_{[0,(\cosh (s)-1) / 2]}(u) \leq k^{+}(u)
$$

and summing over $\gamma \in \Gamma$ :

$$
K^{-}(s, \delta)=\sum_{\gamma \in \Gamma} k^{-}(u(z, \gamma w)) \leq N(s) \leq \sum_{\gamma \in \Gamma} k^{+}(u(z, \gamma w))=K^{+}(s, \delta) .
$$

We want to expand $K^{ \pm}(s, \delta)$ using the pretrace formula. In order to do this we need to prove that Selberg-Harish-Chandra transform of $k^{ \pm}$is an admissible test function.

The Selberg-Harish-Chandra (SHC) transform turns convolutions into products (see [5, p. 323]), so if we denote by $h_{s}$ the SHC transform of $\mathbf{1}_{[0,(\cosh (s)-1) / 2]}$, and by $h^{ \pm}$the SHC transform of $k^{ \pm}$, then we have

$$
h^{ \pm}(t)=\frac{1}{4 \pi \sinh ^{2}(\delta / 2)} h_{s \pm \delta}(t) h_{\delta}(t) .
$$

Denote for simplicity

$$
\tilde{h}_{\delta}(t)=\frac{1}{4 \pi \sinh ^{2}(\delta / 2)} h_{\delta}(t) .
$$

The function $h_{s}(t)$ is explicitely computed in [5, eq. (2.6)] and [20, eq. (2.10)], and is given by

$$
h_{s}(t)=2^{3 / 2} \int_{-s}^{s}(\cosh s-\cosh u)^{1 / 2} e^{i t u} d u .
$$

Observe that $h_{s}(t)$ is a holomorphic function of $t$. Notice also that for every $t \in \mathbb{R}$ and $s>0$ we have the estimate

$$
\left|h_{s}(t)\right| \leq h_{s}(0) \leq s e^{s / 2} .
$$

This will be useful in later estimates for $h(t)$ for $t$ close to 0 .

Lemma 2.4 in [5] shows that $h_{s}(t)$ can be expressed in terms of special functions: for every $s>0$, and every $t \in \mathbb{C}$ such that $i t \notin \mathbb{Z}$, we have

$$
h_{s}(t)=2 \sqrt{2 \pi \sinh s} \Re\left(e^{i t s} \frac{\Gamma(i t)}{\Gamma(3 / 2+i t)} F\left(-\frac{1}{2} ; \frac{3}{2} ; 1-i t ; \frac{1}{\left(1-e^{2 s}\right)}\right)\right),
$$

where $F$ is the Gauss hypergeometric function. Looking at the series expansion of $F$ we can write, for $t \in \mathbb{R}$ and $s>\frac{1}{2} \log 2$,

$$
F\left(-\frac{1}{2} ; \frac{3}{2} ; 1-i t ; \frac{1}{\left(1-e^{2 s}\right)}\right)=1+O\left(e^{-2 s} \min \left\{1, \frac{1}{|t|}\right\}\right) .
$$

Inserting (4.8) in (4.7), using the Taylor expansion of the hyperbolic sine, and Stirling's formula to estimate the quotient of Gamma functions, we obtain the simpler expression

$$
h_{s}(t)=2 \sqrt{\pi} e^{s / 2} \Re\left(e^{i t s} \frac{\Gamma(i t)}{\Gamma(3 / 2+i t)}\right)+O\left(\frac{e^{-3 s / 2}}{|t|\left(1+|t|^{1 / 2}\right)}\right) .
$$


We are also interested in purely imaginary values of $t$ in the interval $[-i / 2, i / 2]$. From [5, Lemma 2.4] and [20, Lemma 2.1] we have, for $s \geq 1$ and $t$ purely imaginary,

$$
h_{s}(t)=\sqrt{2 \pi \sinh s} e^{s|t|} \frac{\Gamma(|t|)}{\Gamma(3 / 2+|t|)}+O\left(\left(1+|t|^{-1}\right) e^{s\left(\frac{1}{2}-|t|\right)}\right) .
$$

For $0 \leq s \leq 1$ and $t \in \mathbb{C}$ we can write instead (see [5, Lemma 2.4 (c)])

$$
h_{s}(t)=2 \pi s^{2} \frac{J_{1}(s t)}{s t} \sqrt{\frac{\sinh s}{s}}+O\left(s^{2} e^{s|\Im t|} \min \left\{s^{2},|t|^{-2}\right\}\right),
$$

where $J_{1}(z)$ is the $J$-Bessel function of order 1 .

4.1. Contribution from the small eigenvalues. In the pretrace formula we split the spectral expansion into the contribution associated to the small eigenvalues and that associated to the rest of the spectrum. We compute now, using the expressions given above for $h_{s}(t)$, the contribution of the small eigenvalues (we also include the case $\lambda=1 / 4)$. We discuss first the contribution coming from the discrete spectrum, and then the contribution coming from the continuous spectrum at $\lambda=1 / 4$.

For the eigenvalue $\lambda_{0}=0$, i.e. $t_{0}=i / 2$, we have a simple formula for $h_{s}(i / 2)$, namely

$$
h_{s}(i / 2)=2 \pi(\cosh s-1) .
$$

This means that we can compute $h^{ \pm}(i / 2)$ directly and obtain

$$
h^{ \pm}(i / 2)=\frac{1}{4 \pi \sinh ^{2}(\delta / 2)} h_{s \pm \delta}(i / 2) h_{\delta}(i / 2)=2 \pi(\cosh s-1)+O\left(\delta e^{s}\right) .
$$

For $\lambda=1 / 4$ we have again a simple expression for $h_{s}(0)$ (see [20, Lemma 2.2]):

$$
h_{s}(0)=4(s+2(\log 2-1)) e^{s / 2}+O\left(e^{-s / 2}\right) .
$$

Using this and (4.11) we can compute the value $h^{ \pm}(0)$. First observe that the function $J_{1}(z)$ verifies

$$
\lim _{z \rightarrow 0} \frac{J_{1}(z)}{z}=\frac{1}{2}
$$

Using the Taylor expansion of the hyperbolic sine for $\delta \ll 1$, we get, in the limit as $t \rightarrow 0$ in (4.11),

$$
\tilde{h}_{\delta}(0)=\frac{1}{4 \pi \sinh ^{2}(\delta / 2)} h_{\delta}(0)=1+O\left(\delta^{2}\right)
$$

Combining (4.13), (4.14), and (4.15), we obtain

$$
h^{ \pm}(0)=h_{s \pm \delta}(0) \tilde{h}_{\delta}(0)=4(s+2(\log 2-1)) e^{s / 2}+O\left(s \delta e^{s / 2}+e^{-s / 2}\right) .
$$

We analyze now the contribution coming from the small eigenvalues $0<\lambda_{j}<1 / 4$. These eigenvalues correspond to $t_{j}$ chosen so that $t_{j} / i \in(0,1 / 2)$. It is important to recall that there is only a finite number of such eigenvalues, which implies that there exists $0<\varepsilon_{\Gamma} \leq 1 / 4$ such that $t_{j} / i \in\left(\varepsilon_{\Gamma}, 1 / 2-\varepsilon_{\Gamma}\right)$. For our analysis we make use of equations (4.10) and (4.11). We can write for $t=t_{j}$ purely imaginary corresponding to a small eigenvalue:

$$
\begin{aligned}
h^{ \pm}(t) & =\left(\sqrt{2 \pi \sinh (s \pm \delta)} e^{(s \pm \delta)|t|} \frac{\Gamma(|t|)}{\Gamma(3 / 2+|t|)}+O\left(\left(1+|t|^{-1}\right) e^{(1 / 2-|t|)(s \pm \delta)}\right)\right) \\
17) & \times\left(\frac{2 \pi \delta^{2}}{4 \pi \sinh ^{2}(\delta / 2)} \frac{J_{1}(\delta t)}{\delta t} \sqrt{\frac{\sinh \delta}{\delta}}+O\left(\frac{\delta^{2} e^{\delta|t|} \min \left\{\delta^{2},|t|^{-2}\right\}}{\sinh ^{2}(\delta / 2)}\right)\right) .
\end{aligned}
$$


Using Taylor approximations in the above formula, for $\delta \ll 1$ and $t=t_{j}$, we can rewrite (4.17) in the more comfortable way

$$
\begin{gathered}
h^{ \pm}(t)=\left[\frac { \Gamma ( | t | ) } { \Gamma ( 3 / 2 + | t | ) } ( \sqrt { \pi } e ^ { s / 2 } + O ( \delta e ^ { s / 2 } + e ^ { - 3 s / 2 } ) ) \left(e^{s|t|}+O\left(\delta e^{s|t|}\right)\right.\right. \\
\left.+O\left(e^{s\left(1 / 2-\varepsilon_{\Gamma}\right)}\right)\right]\left[1+O\left(\delta+\delta^{2} e^{\delta|t|}\right)\right] .
\end{gathered}
$$

Expanding the product we obtain that the contribution from a given small eigenvalue $0<\lambda_{j}<1 / 4$ is given by

$$
h^{ \pm}\left(t_{j}\right)=\sqrt{\pi} \frac{\Gamma\left(\left|t_{j}\right|\right)}{\Gamma\left(3 / 2+\left|t_{j}\right|\right)} e^{s\left(1 / 2+\left|t_{j}\right|\right)}+O\left(\delta e^{s\left(1-\varepsilon_{\Gamma}\right)}+e^{s\left(1 / 2-\varepsilon_{\Gamma}\right)}\right) .
$$

Finally we discuss the contribution coming from the Eisenstein series at $\lambda=1 / 4$. By this contribution we mean the expression

$$
\frac{1}{4 \pi} \sum_{\mathfrak{a}} E_{\mathfrak{a}}(z, 1 / 2) \overline{E_{\mathfrak{a}}(w, 1 / 2)} \int_{-\infty}^{+\infty} h^{ \pm}(t) d t
$$

where the sum runs over the cusps of $\Gamma \backslash \mathbb{H}$. Consider now the integral

$$
\int_{-\infty}^{+\infty} h^{ \pm}(t) d t=\int_{-\infty}^{+\infty} h_{s \pm \delta}(t) d t+\int_{-\infty}^{+\infty} O\left(\left|h_{s \pm \delta}(t)\left(\tilde{h}_{\delta}(t)-1\right)\right|\right) d t .
$$

Using (4.11) and (4.14) we get, for $\delta \ll 1$ and $t \in \mathbb{R}$,

$$
\tilde{h}_{\delta}(t)= \begin{cases}1+O\left(\delta|t|+\delta^{2}\right) & \delta|t|<1 \\ O\left(\frac{1}{(\delta|t|)^{3 / 2}}\right) & \delta|t| \geq 1\end{cases}
$$

By (4.6), (4.7), and (4.8), we have instead

$$
h_{s \pm \delta}(t)=O\left(e^{s / 2} \min \left\{s, \frac{1}{|t|}, \frac{1}{|t|^{3 / 2}}\right\}\right) .
$$

Inserting this in the second integral in (4.19), using (4.20), and splitting the integral to optimize the above estimate, we obtain for $s \geq 1$

$$
\int_{-\infty}^{+\infty}\left|h_{s \pm \delta}(t)\left(\tilde{h}_{\delta}(t)-1\right)\right| d t \ll s \delta^{1 / 2} e^{s / 2} .
$$

Hence we can write

$$
\int_{-\infty}^{+\infty} h^{ \pm}(t) d t=\int_{-\infty}^{+\infty} h_{s \pm \delta}(t) d t+O\left(s \delta^{1 / 2} e^{s / 2}\right)
$$

The average of the function $h_{s}(t)$ can be computed via the Fourier inversion theorem, giving

$$
\int_{\mathbb{R}} h_{s}(t) d t=2 \pi g_{s}(0),
$$

where $g_{s}(u)$ is the Fourier inverse of $h_{s}(t)$ and is given by (see [20, eq. (2.9)])

$$
g_{s}(u)= \begin{cases}2^{3 / 2}(\cosh s-\cosh u)^{1 / 2} & |u| \leq s \\ 0 & \text { otherwise. }\end{cases}
$$

For $s \pm \delta$ and $u=0$ we obtain

$$
2 \pi g_{s \pm \delta}(0)=2^{5 / 2} \pi(\cosh (s \pm \delta)-1)^{1 / 2}=4 \pi e^{s / 2}+O\left(\delta e^{s / 2}+e^{-s / 2}\right) .
$$

This shows that we can write

$$
\int_{\mathbb{R}} h^{ \pm}(t) d t=4 \pi e^{s / 2}+O\left(s \delta^{1 / 2} e^{s / 2}+e^{-s / 2}\right) .
$$


4.1.1. Definition of the main term. We define the complete main term associated to the hyperbolic circle problem as

$$
\begin{aligned}
& \left.M(s, z, w):=\frac{\pi e^{s}}{\operatorname{vol}(\Gamma \backslash \mathbb{H})}+\sqrt{\pi} \sum_{t_{j} \in\left(0, \frac{i}{2}\right)} \frac{\Gamma\left(\left|t_{j}\right|\right)}{\Gamma\left(3 / 2+\left|t_{j}\right|\right)} e^{s\left(1 / 2+\left|t_{j}\right|\right)} \phi_{j}(z) \overline{\phi_{j}(w}\right) \\
& \left.\left.+4(s+2(\log 2-1)) e^{s / 2} \sum_{t_{j}=0} \phi_{j}(z) \overline{\phi_{j}(w}\right)+e^{s / 2} \sum_{\mathfrak{a}} E_{\mathfrak{a}}(z, 1 / 2) \overline{E_{\mathfrak{a}}(w, 1 / 2}\right) .
\end{aligned}
$$

Denote by $M^{ \pm}(s, \delta)$ the main term associated to $K^{ \pm}(s, \delta)$, defined as the contribution coming from the eigenvalues $\lambda_{j} \leq 1 / 4$ (together with the contribution from the continuous spectrum at $\lambda=1 / 4$ ) for the kernels $K^{ \pm}$. In other words,

$$
\begin{aligned}
M^{ \pm}(s, \delta) & :=\sum_{t_{j} \in\left[0, \frac{i}{2}\right]} h^{ \pm}\left(t_{j}\right) \phi_{j}(z) \overline{\phi_{j}(w)} \\
& \left.+\frac{1}{4 \pi} \sum_{\mathfrak{a}} E_{\mathfrak{a}}(z, 1 / 2) \overline{E_{\mathfrak{a}}(w, 1 / 2}\right) \int_{\mathbb{R}} h^{ \pm}(t) d t
\end{aligned}
$$

Then we can summarize equations (4.12), (4.16), (4.18), and (4.22) by saying that

$$
M^{ \pm}(s, \delta, z, w)=M(s)+O\left(\delta e^{s}+s \delta^{1 / 2} e^{s / 2}+e^{s\left(1 / 2-\varepsilon_{\Gamma}\right)}\right)
$$

(in order to simplify notation we have omitted the $z, w$ dependence of $M$ and $M^{ \pm}$).

4.2. Proof of Theorem 1.15. From the inequality (4.4) we have

$$
|E(s, z, w)| \leq \max \left\{\left|K^{-}(s, \delta)-M(s)\right|,\left|K^{+}(s, \delta)-M(s)\right|\right\} .
$$

Squaring and integrating we see that in order to prove Theorem 1.15 it suffices to prove that for some $\delta=\delta(T)$ we have

$$
\int_{T}^{T+1}\left|\frac{K^{ \pm}(s, \delta)-M(s)}{e^{s / 2}}\right|^{2} d s \ll T .
$$

Using (4.25) we have

$$
K^{ \pm}(s, \delta)-M(s)=K^{ \pm}(s, \delta)-M^{ \pm}(s)+O\left(\delta e^{s}+s \delta^{1 / 2} e^{s / 2}+e^{s\left(1 / 2-\varepsilon_{\Gamma}\right)}\right) .
$$

We will show later that the choice $\delta(T)=e^{-T / 2}$ is admissible. This leads to

$$
\int_{T}^{T+1}\left|\frac{K^{ \pm}(s, \delta)-M(s)}{e^{s / 2}}\right|^{2} d s=H(T)+O\left(H(T)^{1 / 2}+1\right),
$$

where

$$
H(T):=\int_{T}^{T+1}\left|\frac{K^{ \pm}(s, \delta)-M^{ \pm}(s)}{e^{s / 2}}\right|^{2} d s .
$$

The problem reduces thus to show that $H(T) \ll T$, which we can prove using the pretrace formula. Before proceeding to do so, though, we insert a weight function in (4.29), which turns out useful for multiple integration by parts. Let $\psi(s) \in C_{c}^{\infty}(\mathbb{R})$, with $\operatorname{supp}(\psi) \subseteq[-1 / 2,3 / 2]$, such that $0 \leq \psi \leq 1$ and $\psi(s)=1$ for $s \in[0,1]$. Define, for $T \geq 0, \psi_{T}(s)=\psi(s-T)$. Then we have the inequality

$$
H(T) \leq \int_{\mathbb{R}}\left|\frac{K^{ \pm}(s, \delta)-M^{ \pm}(s)}{e^{s / 2}}\right|^{2} \psi_{T}(s) d s,
$$

and we give bounds on this last integral. The pretrace formula applied to the function $K^{ \pm}(s, \delta)$ gives

$$
K^{ \pm}(s, \delta)-M^{ \pm}(s, \delta)=\sum_{t_{j}>0} h^{ \pm}\left(t_{j}\right) \phi_{j}(z) \overline{\phi_{j}(w)}+\frac{1}{4 \pi} \sum_{\mathfrak{a}} \int_{-\infty}^{+\infty} h^{ \pm}(t) E_{\mathfrak{a}}(t) d t
$$


where in order to shorten the notation we have written

$$
\left.\left.E_{\mathfrak{a}}(t)=E_{\mathfrak{a}}(z, 1 / 2+i t) \overline{E_{\mathfrak{a}}(w, 1 / 2+i t}\right)-E_{\mathfrak{a}}(z, 1 / 2) \overline{E_{\mathfrak{a}}(w, 1 / 2}\right) .
$$

The series and the integral in (4.31) are absolutely convergent, as for $\delta>0$ we have $h^{ \pm}(t) \ll t^{-3}$ (this follows from the definition (4.5) of $h^{ \pm}(t)$ and the estimates (4.20) and (4.21)). We also use, for fixed $z \in \mathbb{H}$, the following standard inequality (for a proof see $[16, \S 13.2])$ :

$$
\sum_{T \leq t_{j} \leq T+1}\left|\phi_{j}(z)\right|^{2}+\frac{1}{4 \pi} \sum_{\mathfrak{a}} \int_{T}^{T+1}\left|E_{\mathfrak{a}}(z, 1 / 2+i t)\right|^{2} d t \ll T .
$$

In order to give upper bounds on the second moment of $K^{ \pm}(s, \delta)-M^{ \pm}(s, \delta)$, it suffices to estimate separately the square of the series and the square of the integrals in (4.31). We prove a lemma that is useful for this purpose.

Lemma 4.1. Let $T \gg 1$ and $0<\delta \ll 1$. Let $t_{1}, t_{2} \in \mathbb{R}, t_{1}, t_{2} \neq 0$. Then

$$
\int_{\mathbb{R}} \frac{\left.h_{s \pm \delta}\left(t_{1}\right) \overline{h_{s \pm \delta}\left(t_{2}\right.}\right)}{e^{s}} \psi_{T}(s) d s \ll \frac{g\left(t_{1}\right) g\left(t_{2}\right)}{1+\left|t_{1}-t_{2}\right|^{2}}+\frac{g\left(t_{1}\right) g\left(t_{2}\right)}{1+\left|t_{1}+t_{2}\right|^{2}}+\frac{g\left(t_{1}\right) g\left(t_{2}\right)}{e^{2 T}}
$$

where $g(t)=(|t|(1+\sqrt{|t|}))^{-1}$.

Proof. From (4.9) we can write

$$
\begin{aligned}
\frac{h_{s \pm \delta}\left(t_{1}\right) \overline{h_{s \pm \delta}\left(t_{2}\right)}}{e^{s}} & =\pi e^{ \pm \delta} \Re\left(e^{i(s \pm \delta)\left(t_{1}-t_{2}\right)} G\left(t_{1}\right) \overline{G\left(t_{2}\right)}\right) \\
& +\pi e^{ \pm \delta} \Re\left(e^{i(s \pm \delta)\left(t_{1}+t_{2}\right)} G\left(t_{1}\right) G\left(t_{2}\right)\right)+O\left(g\left(t_{1}\right) g\left(t_{2}\right) e^{-2 s}\right),
\end{aligned}
$$

where $G(t)=\Gamma(i t) / \Gamma(3 / 2+i t)$, and $G(t) \ll g(t)$. After multiplying by $\psi_{T}(s)$, the integral over $s$ can be estimated by taking the absolute value inside the integral, or integrating by parts and then bounding the result. The two different bounds lead to $(4.33)$.

4.2.1. Discrete spectrum. Consider the series in (4.31). Taking absolute value and squaring gives a double sum, which we then multiply by $\psi_{T}(s)$ and integrate over $s$. For simplicity we assume that $z=w$, but the same argument works for $z \neq w$. We claim that the following holds:

$$
\sum_{t_{j}, t_{\ell}>0}\left|\phi_{j}(z)\right|^{2}\left|\phi_{\ell}(z)\right|^{2} \int_{\mathbb{R}} \frac{\left.h^{ \pm}\left(t_{j}\right) \overline{h^{ \pm}\left(t_{\ell}\right.}\right)}{e^{s}} \psi_{T}(s) d s \ll \log \left(\delta^{-1}\right)+\delta^{-1} e^{-2 T}+1 .
$$

By symmetry of the estimate (4.33) in Lemma 4.1, and positivity of the integral in (4.35) for $t_{j}=t_{\ell}$, it is sufficient to consider only the case when $t_{\ell} \geq t_{j}$.

We follow here a classical argument to analyse the sum (see e.g. [8, 9]). We consider the part of the series in (4.35) where $t_{j}$ and $t_{\ell}$ are close to each other. Using (4.20), (4.32), and Lemma 4.1, we have the following estimates:

$$
\begin{aligned}
& \sum_{\substack{t_{j}>0 \\
t_{j} \leq t_{\ell}<t_{j}+1}}\left|\phi_{j}(z)\right|^{2}\left|\phi_{\ell}(z)\right|^{2} \int_{\mathbb{R}} \frac{\left.h^{ \pm}\left(t_{j}\right) \overline{h^{ \pm}\left(t_{\ell}\right.}\right)}{e^{s}} \psi_{T}(s) d s \\
\ll & \sum_{\substack{t_{j}<\delta^{-1} \\
t_{j} \leq t_{\ell}<t_{j}+1}} \frac{\left|\phi_{j}(z)\right|^{2}\left|\phi_{\ell}(z)\right|^{2}}{\left(t_{j} t_{\ell}\right)^{3 / 2}}+\sum_{\substack{t_{j} \geq \delta^{-1} \\
t_{j} \leq t_{\ell}<t_{j}+1}} \frac{\left|\phi_{j}(z)\right|^{2}\left|\phi_{\ell}(z)\right|^{2}}{\left(\delta t_{j} t_{\ell}\right)^{3}} \\
\ll & \sum_{t_{j}<\delta^{-1}} \frac{\left|\phi_{j}(z)\right|^{2}}{t_{j}^{2}}+\frac{1}{\delta^{3}} \sum_{t_{j} \geq \delta^{-1}} \frac{\left|\phi_{j}(z)\right|^{2}}{t_{j}^{5}} \ll \log \delta^{-1}+1 .
\end{aligned}
$$


This shows that a neighbourhood of the diagonal $t_{j}=t_{\ell}$ (of width 1 ) gives a contribution of the order $O\left(\log \delta^{-1}+1\right)$. Similarly we can estimate

$$
\begin{aligned}
& \sum_{\substack{t_{j} \geq \delta^{-1} \\
t_{\ell} \geq t_{j}+1}}\left|\phi_{j}(z)\right|^{2}\left|\phi_{\ell}(z)\right|^{2} \int_{\mathbb{R}} \frac{\left.h^{ \pm}\left(t_{j}\right) \overline{h^{ \pm}\left(t_{\ell}\right.}\right)}{e^{s}} \psi_{T}(s) d s \\
& \ll \frac{1}{\delta^{3}} \sum_{t_{j} \geq \delta^{-1}} \frac{\left|\phi_{j}(z)\right|^{2}}{t_{j}^{3}} \sum_{t_{\ell} \geq t_{j}+1} \frac{\left|\phi_{\ell}(z)\right|^{2}}{t_{\ell}^{3}}\left(\frac{1}{\left|t_{\ell}-t_{j}\right|^{2}}+e^{-2 T}\right),
\end{aligned}
$$

and using now a unit intervals decomposition we obtain the bound

$$
\begin{aligned}
& \ll \frac{1}{\delta^{3}} \sum_{t_{j} \geq \delta^{-1}} \frac{\left|\phi_{j}(z)\right|^{2}}{t_{j}^{3}}\left(\sum_{n=1}^{\infty} \sum_{n \leq t_{\ell}-t_{j} \leq n+1} \frac{\left|\phi_{\ell}(z)\right|^{2}}{t_{\ell}^{3}\left|t_{\ell}-t_{j}\right|^{2}}+\frac{e^{-2 T}}{t_{j}}\right) \\
& \ll \frac{1}{\delta^{3}} \sum_{t_{j} \geq \delta^{-1}} \frac{\left|\phi_{j}(z)\right|^{2}}{t_{j}^{3}}\left(\sum_{n=1}^{\infty} \frac{1}{n^{2}\left(t_{j}+n\right)^{2}}+\frac{e^{-2 T}}{t_{j}}\right) \\
& \ll \frac{1}{\delta^{3}} \sum_{t_{j} \geq \delta^{-1}} \frac{\left|\phi_{j}(z)\right|^{2}}{t_{j}^{5}}+\frac{\left|\phi_{j}(z)\right|^{2}}{t_{j}^{4}} e^{-2 T} \ll 1+\delta^{-1} e^{-2 T} .
\end{aligned}
$$

This shows that the "tail" of the double series in (4.35) gives a contribution of the order $O\left(1+\delta^{-1} e^{-2 T}\right)$. Finally, the sum for $t_{j}<\delta^{-1}$ and $t_{\ell}$ large can be bounded as follows:

$$
\begin{aligned}
& \sum_{\substack{t_{j}<\delta^{-1} \\
t_{\ell} \geq t_{j}+1}}\left|\phi_{j}(z)\right|^{2}\left|\phi_{\ell}(z)\right|^{2} \int_{\mathbb{R}} \frac{\left.h^{ \pm}\left(t_{j}\right) \overline{h^{ \pm}\left(t_{\ell}\right.}\right)}{e^{s}} \psi_{T}(s) d s \\
& \ll \sum_{t_{j}<\delta^{-1}} \frac{\left|\phi_{j}(z)\right|^{2}}{t_{j}^{3 / 2}}\left(\sum_{t_{\ell} \geq t_{j}+1} \frac{\left|\phi_{\ell}(z)\right|^{2}}{t_{\ell}^{3} / 2\left|t_{\ell}-t_{j}\right|^{2}}+\right. \\
& \left.+\sum_{t_{j}+1 \leq t_{\ell} \leq \delta^{-1}} \frac{\left|\phi_{\ell}(z)\right|^{2}}{t_{\ell}^{3 / 2}} e^{-2 T}+\frac{1}{\delta^{3 / 2}} \sum_{t_{\ell} \geq \delta^{-1}} \frac{\left|\phi_{\ell}(z)\right|^{2}}{t_{\ell}^{3}} e^{-2 T}\right),
\end{aligned}
$$

and again a unit intervals decomposition gives

$$
\begin{aligned}
& \ll \sum_{t_{j}<\delta^{-1}} \frac{\left|\phi_{j}(z)\right|^{2}}{t_{j}^{3 / 2}}\left(\sum_{n=1}^{\infty} \frac{1}{n^{2}\left(t_{j}+n\right)^{1 / 2}}+\delta^{-1 / 2} e^{-2 T}\right) \\
& \ll \sum_{t_{j}<\delta^{-1}} \frac{\left|\phi_{j}(z)\right|^{2}}{t_{j}^{2}}+\delta^{-1} e^{-2 T} \ll \log \delta^{-1}+\delta^{-1} e^{-2 T} .
\end{aligned}
$$

Adding together (4.36), (4.37), and (4.38), we conclude that (4.35) holds, as claimed.

4.2.2. Continuous spectrum. Consider, for a fixed cusp a, the associated integral in (4.31). As in the case of the discrete spectrum, we take absolute value, square, multiply by $\psi_{T}(s)$, and then integrate over $s$. Again we assume that $z=w$, but the same argument works for $z \neq w$. We claim that the following holds:

$$
\int_{\mathbb{R}}\left|\int_{-\infty}^{+\infty} \frac{h^{ \pm}(t)}{e^{s / 2}} E_{\mathfrak{a}}(t) d t\right|^{2} \psi_{T}(s) d s \ll \log \delta^{-1}+1+\delta^{-1} e^{-2 T} .
$$

Since both $h^{ \pm}(t)$ and $E_{\mathfrak{a}}(t)$ are even in $t$, we can restrict the domain of integration (for the inner integral) to the positive real axis $[0,+\infty)$. So we need to prove

$$
\int_{\mathbb{R}} \int_{0}^{\infty} \int_{0}^{\infty} E_{\mathfrak{a}}\left(t_{1}\right) E_{\mathfrak{a}}\left(t_{2}\right) \frac{h^{ \pm}\left(t_{1}\right) \overline{h^{ \pm}\left(t_{2}\right)}}{e^{s}} \psi_{T}(s) d t_{1} d t_{2} d s \ll \log \delta^{-1}+1+\delta^{-1} e^{-2 T}
$$


Since the integrals are absolutely convergent, we can interchange the order of integration. Moreover, by symmetry and positivity of the integral for $t_{1}=t_{2}$, it suffices to discuss the integral over $t_{2} \geq t_{1}$.

The analysis is similar to the case of the discrete spectrum, except for $t_{1}, t_{2}$ close to zero, when we exploit the fact that the Eisenstein series is regular at $1 / 2$ to bound $E_{\mathfrak{a}}(t)=O(t)$. Using this together with (4.20), (4.32), and Lemma 4.1, we can bound a unit neighbourhood of the diagonal $t_{1}=t_{2}$ by

$$
\int_{0}^{\infty} \int_{t_{1}}^{t_{1}+1} E_{\mathfrak{a}}\left(t_{1}\right) E_{\mathfrak{a}}\left(t_{2}\right) \int_{\mathbb{R}} \frac{\left.h^{ \pm}\left(t_{1}\right) \overline{h^{ \pm}\left(t_{2}\right.}\right)}{e^{s}} \psi_{T}(s) d s d t_{2} d t_{1} \ll \log \left(\delta^{-1}\right)+1 .
$$

The tail of the integral can be bounded by

$$
\int_{\delta^{-1}}^{+\infty} \int_{t_{1}+1}^{+\infty} E_{\mathfrak{a}}\left(t_{1}\right) E_{\mathfrak{a}}\left(t_{2}\right) \int_{\mathbb{R}} \frac{\left.h^{ \pm}\left(t_{1}\right) \overline{h^{ \pm}\left(t_{2}\right.}\right)}{e^{s}} \psi_{T}(s) d s d t_{2} d t_{1} \ll \delta^{-1} e^{-2 T}+1 .
$$

Finally, the range with $t_{1}<\delta^{-1}$ and $t_{2}$ large is bounded similarly by

$$
\ll \log \left(\delta^{-1}\right)+\delta^{-1} e^{-2 T}+1 \text {. }
$$

Summing (4.41), (4.42), and (4.43), we conclude that (4.39) holds. Inserting (4.35) and (4.39) into (4.30), and choosing $\delta=e^{-T / 2}$, we see that we have proved the bound

$$
H(T) \ll T,
$$

as we wanted. This proves Theorem 1.15.

\section{INTEGRATED REMAINDERS}

In this section we prove Theorem 1.19. We start by noting that for $\Gamma$ cocompact the function $N(s, z, w)$ is uniformly bounded in $z, w$ and hence square-integrable (see e.g. [22, Thm. 6.1]) and [6, p. 278]). By Parseval's theorem we get the expansion

$$
G_{1}(s, z)=\sum_{t_{j}>0} \frac{h_{s}\left(t_{j}\right)^{2}}{e^{s}}\left|\phi_{j}(z)\right|^{2}+\sum_{t_{j} \in[0, i / 2]} f_{s}\left(t_{j}\right)^{2}\left|\phi_{j}(z)\right|^{2},
$$

where $f_{s}\left(t_{j}\right)$ is defined (recall (4.23)) for $t_{j} \in[0, i / 2]$ by

$$
\begin{aligned}
f_{s}(i / 2) & =\frac{1}{e^{s / 2}}\left(h_{s}(i / 2)-\frac{\pi e^{s}}{\operatorname{vol}(\Gamma \backslash \mathbb{H})}\right), \\
f_{s}\left(t_{j}\right) & =\frac{1}{e^{s / 2}}\left(h_{s}\left(t_{j}\right)-\sqrt{\pi} \frac{\Gamma\left(\left|t_{j}\right|\right)}{\Gamma\left(3 / 2+\left|t_{j}\right|\right)} e^{s\left(1 / 2+\left|t_{j}\right|\right)}\right), t_{j} \in(0, i / 2), \\
f_{s}(0) & =\frac{1}{e^{s / 2}}\left(h_{s}(0)-4(s+2(\log 2-1)) e^{s / 2}\right)
\end{aligned}
$$

and it satisfies $f_{s}\left(t_{j}\right)=O\left(e^{-\varepsilon_{\Gamma} s}\right)$ for every $t_{j} \in[0, i / 2]$, for some $\varepsilon_{\Gamma}>0$ (this follows from the discussion on the small eigenvalues in section 4.1). We can therefore write

$$
G_{1}(s, z)=C_{1}+2 \Re\left(\sum_{0<t_{j} \leq X} \frac{\pi \Gamma\left(i t_{j}\right)^{2}\left|\phi_{j}(z)\right|^{2}}{\Gamma\left(3 / 2+i t_{j}\right)^{2}} e^{2 i t_{j} s}\right)+O\left(e^{-\varepsilon_{\Gamma} s}+\frac{1}{X}\right)
$$

with

$$
C_{1}=2 \pi \sum_{t_{j}>0} \frac{\left|\Gamma\left(i t_{j}\right)\right|^{2}}{\left|\Gamma\left(3 / 2+i t_{j}\right)\right|^{2}}\left|\phi_{j}(z)\right|^{2} .
$$

The coefficients in (5.1) satisfy (by (4.9) and (4.32))

$$
\sum_{T \leq 2 t_{j} \leq T+1} \frac{\left|\Gamma\left(i t_{j}\right)\right|^{2}}{\left|\Gamma\left(3 / 2+i t_{j}\right)\right|^{2}}\left|\phi_{j}(z)\right|^{2} \ll \frac{1}{T^{2}} .
$$


In particular this means that $G_{1}(s, z)$ is bounded in $s$, because

$$
\left|G_{1}(s, z)\right| \ll C_{1}+\sum_{t_{j}>0} \frac{\left|\phi_{j}(z)\right|^{2}}{t_{j}^{3}}+e^{-\varepsilon_{\Gamma} s} \ll_{z} 1 .
$$

The function $G_{1}-C_{1}$ is of the form (1.7), satisfies (1.9) with $\beta=2$, and, choosing $X(Y)=e^{Y}$, it satisfies (1.8) for every $p \geq 1$. Hence we can apply Theorem 1.3 and infer the existence of all the moments, and Theorem 1.10 to infer the existence of a limiting distribution $\tilde{\mu}_{1}$. By Corollary 1.14 , the moments of $G_{1}-C_{1}$ coincide with the moments of $\tilde{\mu}_{1}$. Shifting $G_{1}-C_{1}$ and the measure $\tilde{\mu}_{1}$ by adding back $C_{1}$ we obtain the Theorem for $G_{1}$.

Consider now the function $G_{2}(s)$. In this case we have the expansion

$$
G_{2}(s)=\sum_{t_{j}>0} \frac{h_{s}\left(t_{j}\right)^{2}}{e^{s}}+\sum_{t_{j} \in[0, i / 2]} f_{s}\left(t_{j}\right)^{2},
$$

and therefore we can write

$$
G_{2}(s)=C_{2}+2 \Re\left(\sum_{0<t_{j} \leq X} \frac{\pi \Gamma\left(i t_{j}\right)^{2}}{\Gamma\left(3 / 2+i t_{j}\right)^{2}} e^{2 i t_{j} s}\right)+O\left(e^{-\varepsilon_{\Gamma} s}+\frac{1}{X}\right)
$$

with

$$
C_{2}=2 \pi \sum_{t_{j}>0} \frac{\left|\Gamma\left(i t_{j}\right)\right|^{2}}{\left|\Gamma\left(3 / 2+i t_{j}\right)\right|^{2}}
$$

Using this time the estimate [25, Th. 7.3] on the distribution of the eigenvalues

$$
\sum_{T \leq 2 t_{j} \leq T+1} 1 \ll T
$$

we can write

$$
\sum_{T \leq 2 t_{j} \leq T+1} \frac{\Gamma\left(i t_{j}\right)^{2}}{\Gamma\left(3 / 2+i t_{j}\right)^{2}} \ll \frac{1}{T^{2}}
$$

and choosing again $X=e^{Y}$ we see that $G_{2}-C_{2}$ is of the form (1.7), satisfies (1.9) with $\beta=2$, and (1.8) for every $p \geq 1$. Applying Theorem 1.3, Theorem 1.10, and Corollary 1.14, we conclude the proof for $G_{2}$.

Finally consider the function $G_{3}(s, z)$. The function $e^{-s / 2} \int_{0}^{s} N(x, z, z) d x$ is an automorphic kernel associated to the function $k_{s}^{*}(u)=e^{-s / 2} \int_{0}^{s} k_{x}(u) d x$, where $k_{x}(u)=\mathbf{1}_{[0,(\cosh x-1) / 2)]}(u)$. The Selberg-Harish-Chandra transform $h_{s}^{*}$ of $k_{s}^{*}$ is given by

$$
h_{s}^{*}(t)=\frac{1}{e^{s / 2}} \int_{0}^{s} h_{x}(t) d x
$$

and can be analysed with analogous computations to those of section 4 . We claim that $h^{*}$ is an admissible function in the pretrace formula. First observe that for the small eigenvalues we can write

$$
h_{s}^{*}(i / 2)=\frac{1}{e^{s / 2}} \int_{0}^{s}\left(\pi e^{x}+O(1)\right) d x=\pi e^{s / 2}+O\left(s e^{-s / 2}\right) .
$$

Similarly we have

$$
h_{s}^{*}(0)=8(s+2(\log 2-1))+O\left(e^{-s / 2}\right),
$$

and, for $t_{j} \in(0, i / 2)$,

$$
h_{s}^{*}\left(t_{j}\right)=\sqrt{\pi} \frac{\Gamma\left(\left|t_{j}\right|\right)}{\left(1 / 2+\left|t_{j}\right|\right) \Gamma\left(3 / 2+\left|t_{j}\right|\right)} e^{s\left|t_{j}\right|}+O\left(e^{-\varepsilon_{\Gamma} s}\right)
$$


for some $0<\varepsilon_{\Gamma}<1 / 4$. For $t_{j}$ real and positive we use a representation for $h_{x}(t)$ that is more suitable for integration in $x$. The expression can be found in the proof of [20, Lemma 2.5], and gives

$$
h_{x}\left(t_{j}\right)=2 \Re\left(I\left(x, t_{j}\right) e^{x\left(1 / 2+i t_{j}\right)}\right),
$$

where

$$
I\left(x, t_{j}\right)=-2 i \int_{0}^{\infty}\left(1-e^{i v}\right)^{1 / 2}\left(1-e^{-2 x-i v}\right)^{1 / 2} e^{-t_{j} v} d v .
$$

Integrating in $x$ we can write

$$
\int_{0}^{s} h_{x}\left(t_{j}\right) d x=2 \Re\left(\int_{0}^{s} I\left(x, t_{j}\right) e^{x\left(1 / 2+i t_{j}\right)} d x\right) .
$$

Moving the contour of integration to two vertical lines above 0 and $s$ in the complex plane, we obtain

$$
\begin{aligned}
& \int_{0}^{s} I\left(x, t_{j}\right) e^{x\left(1 / 2+i t_{j}\right)} d x \\
& =2 \int_{0}^{\infty} \int_{0}^{\infty}\left(1-e^{i v}\right)^{1 / 2}\left(1-e^{-2 i \lambda-i v}\right)^{1 / 2} e^{-t_{j} v} e^{i \lambda / 2-\lambda t_{j}} d v d \lambda \\
& -2 e^{s\left(1 / 2+i t_{j}\right)} \int_{0}^{\infty} \int_{0}^{\infty}\left(1-e^{i v}\right)^{1 / 2}\left(1-e^{-2 s-2 i \lambda-i v}\right)^{1 / 2} e^{-t_{j} v} e^{i \lambda / 2-\lambda t_{j}} d v d \lambda .
\end{aligned}
$$

Isolating the oscillation $e^{i s t_{j}}$, and using the bound $\left|1-e^{i v}\right| \ll \min (1, v)$ to bound the other terms, we conclude that

$$
h_{s}^{*}\left(t_{j}\right)=2 \Re\left(A\left(t_{j}\right) e^{i s t_{j}}\right)+O\left(\frac{1}{t_{j}^{5 / 2}}\right),
$$

with

$$
A\left(t_{j}\right)=-2 \int_{0}^{\infty} \int_{0}^{\infty}\left(1-e^{i v}\right)^{1 / 2} e^{-t_{j} v} e^{i \lambda / 2-\lambda t_{j}} d v d \lambda=\frac{i \sqrt{\pi} \Gamma\left(i t_{j}\right)}{(1 / 2-i t) \Gamma\left(3 / 2+i t_{j}\right)} .
$$

Since $A\left(t_{j}\right) \ll t_{j}^{-5 / 2}$, we infer that $h_{s}^{*}$ is an admissible test function in the pretrace formula. Observing moreover that the main terms that appear in the integration of the small eigenvalues correspond to the integration of the terms defining $M(s, z, z)$, we can write for $X \gg 1$

$$
G_{3}(s, z)=2 \Re\left(\sum_{0<t_{j} \leq X} A\left(t_{j}\right)\left|\phi_{j}(z)\right|^{2} e^{i s t_{j}}\right)+O\left(e^{-\varepsilon_{\Gamma} s}+\frac{1}{X^{1 / 2}}\right) .
$$

This shows that $G_{3}(s, z)$ is of the form (1.7), and its coefficients $A\left(t_{j}\right)\left|\phi_{j}(z)\right|^{2}$ satisfy (1.9) with $\beta=3 / 2$. Choosing $X=e^{Y}, G_{3}(s, z)$ satisfies (1.8) for every $p \geq 1$. Applying Theorem 1.3, Theorem 1.10, and Corollary 1.14, we conclude the proof for $G_{3}$.

\section{ACKNOWLEDGMENTS}

I would like to thank Morten S. Risager and Yiannis Petridis for their precious comments on earlier versions of the paper. Thanks also go to the anonymus referee, whose comments helped to improve significantly the exposition of the paper. This work was supported by a Sapere Aude grant from The Danish Council for Independent Research (Grant-id:0602-02161B). 


\section{REFERENCES}

[1] A. Akbary, N. Ng, and M. Shahabi, Limiting Distributions of the Classical Error Terms of Prime Number Theory, Q. J. Math. 65 (2014), no. 3, 743-780. 1, 2, 3, 4, 5

[2] P. M. Bleher, Z. Cheng, F. J. Dyson, and J. L. Lebowitz, Distribution of the error term for the number of lattice points inside a shifted circle, Comm. Math. Phys. 154 (1993), no. 3, 433-469. 4

[3] F. Chamizo, Topics in Analytic Number Theory, Ph.D. thesis, Universidad Autonoma de Madrid, 1994. 5

[4] F. Chamizo, The large sieve in Riemann surfaces, Acta Arith. 77 (1996), no. 4, 303-313. 5

[5] F. Chamizo, Some applications of large sieve in Riemann surfaces, Acta Arith. 77 (1996), no. 4, 315-337. 5, 12, 13

[6] I. Chavel, Eigenvalues in Riemannian geometry, Pure and Applied Mathematics, vol. 115, Academic Press, Inc., Orlando, FL, 1984. 18

[7] G. Cherubini and M. S. Risager, On the variance of the error term in the hyperbolic circle problem, Rev. Mat. Iberoamericana 34 (2018), no. 2, 655-685. 6

[8] H. Cramér, Ein Mittelwertsatz in der Primzahltheorie, Math. Z. 12 (1922), no. 1, 147-153. 1,16

[9] H. Cramér, Über zwei Sätze des Herrn G. H. Hardy, Math. Z. 15 (1922), no. 1, 201-210. 1, 16

[10] H. Davenport, Multiplicative number theory, third ed., Graduate Texts in Mathematics, vol. 74, Springer-Verlag, New York, 2000. 2, 3

[11] D. Fiorilli, Elliptic Curves of Unbounded Rank and Chebyshev's Bias, Int. Math. Res. Not. 2014 (2014), no. 18, 4997-5024. 10

[12] D. R. Heath-Brown, The distribution and moments of the error term in the Dirichlet divisor problem, Acta Arith. 60 (1991), no. 4, 389-415. 1, 4

[13] D. R. Heat-Brown, The Dirichlet divisor problem, Advances in number theory (Kingston, ON, 1991), Oxford Sci. Publ., Oxford Univ. Press, New York, 1993, pp. 31-35. 1

[14] R. Hill and L. Parnovski, The variance of the hyperbolic lattice point counting function, Russ. J. Math. Phys. 12 (2005), no. 4, 472-482. 6

[15] A. Ivić, The Riemann zeta function, A Wiley-Interscience Publication. John Wiley and Sons, Inc., New York, 1985. xvi+517 pp. 2

[16] H. Iwaniec, Spectral methods of automorphic forms, second ed., Graduate Studies in Mathematics, vol. 53, Amer. Math. Soc., Providence, RI; Rev. Mat. Iberoam., Madrid, 2002. 11, 16

[17] H. Iwaniec and E. Kowalski, Analytic number theory, Amer. Math. Soc. Colloquium Publications, 53. Amer. Math. Soc., Providence, RI, 2004. xii+615 pp. 9

[18] H. L. Montgomery, The zeta function and prime numbers, Proceedings of the Queen's Number Theory Conference, 1979 (Kingston, Ont., 1979), Queen's Papers in Pure and Appl. Math., vol. 54, Queen's Univ., Kingston, Ont., 1980, pp. 1-31. 5

[19] H. L. Montgomery and Andrew M. Odlyzko, Large deviations of sums of independent random variables, Acta Arith. 49 (1987), no. 4, 427-434. 5

[20] R. Phillips and Z. Rudnick, The Circle Problem in the Hyperbolic Plane, J. Funct. Anal. 121 (1994), no. 1, 78-116. 5, 6, 12, 13, 14, 20

[21] M. Rubinstein and P. Sarnak, Chebyshev's bias, Exp. Math. 3 (1994), no. 3, 173-197. 4, 5, 9,10

[22] A. Selberg, Harmonic Analysis, Atle Selberg collected papers Vol 1, Springer (1955), 628-674. 18

[23] E. C. Titchmarsh, The theory of the Riemann zeta-function, second ed., The Clarendon Press, Oxford University Press, New York, 1986. 2

[24] K.-M. Tsang, Higher-Power Moments of $(x), E(t)$ and P(x), Proc. Lond. Math. Soc. s3-65 (1992), no. 1, 65-84. 1

[25] A. B. Venkov, Spectral theory of automorphic functions and its applications, Mathematics and its Applications (Soviet Series), vol. 51, Kluwer Academic Publishers Group, Dordrecht, 1990, Translated from the Russian by N. B. Lebedinskaya. 19

[26] A. Wintner, On the Asymptotic Distribution of the Remainder Term of the Prime-Number Theorem, Amer. J. Math. 57 (1935), no. 3, 534-538. 1

[27] W. Wolfe, The asymptotic distribution of lattice points in hyperbolic space, J. Funct. Anal. 31 (1979), no. 3, 333-340. 6

Dipartimento di Matematica, Università di Genova, Via Dodecaneso 35, 16146 Genoa ITALY

E-mail address: cherubini@dima.unige.it 